Accepted by AJ

\title{
Further evaluation of bootstrap resampling as a tool for radio-interferometric imaging fidelity assessment
}

\author{
Athol Kemball \\ Department of Astronomy and Institute for Advanced Computing Applications and \\ Technologies/NCSA, University of Illinois at Urbana-Champaign, 1002 W. Green Street, \\ Urbana, IL 61801 \\ Adam Martinsek \\ Department of Statistics, University of Illinois at Urbana-Champaign, $725 \mathrm{~S}$. Wright \\ Street, Champaign, IL 61820 \\ Modhurita Mitra and Hsin-Fang Chiang \\ Department of Astronomy, University of Illinois at Urbana-Champaign, 1002 W. Green \\ Street, Urbana, IL 61801
}

\begin{abstract}
We report on a broader evaluation of statistical bootstrap resampling methods as a tool for pixel-level calibration and imaging fidelity assessment in radio interferometry. Pixel-level imaging fidelity assessment is a challenging problem, important for the value it holds in robust scientific interpretation of interferometric images, enhancement of automated pipeline reduction systems needed to broaden the user community for these instruments, and understanding leadingedge direction-dependent calibration and imaging challenges for future telescopes such as the Square Kilometer Array. This new computational approach is now possible because of advances in statistical resampling for data with long-range dependence and the available performance of contemporary high-performance computing resources. We expand our earlier numerical evaluation to span a broader domain subset in simulated image fidelity and source brightness distribution morphologies. As before, we evaluate the statistical performance of the bootstrap resampling methods against direct Monte Carlo simulation. We find both model-based and subsample bootstrap methods to continue to show significant promise for the challenging problem of interferometric imaging fidelity
\end{abstract}


assessment, when evaluated over the broader domain subset. We report on their measured statistical performance and guidelines for their use and application in practice. We also examine the performance of the underlying polarization selfcalibration algorithm used in this study over a range of parallactic angle coverage.

Subject headings: techniques: image processing — methods: statistical — techniques: interferometric — techniques: polarimetric

\section{Introduction}

Radio-interferometric image formation requires a solution for both the source brightness distribution over the image field and the interferometric array instrumental and signal propagation effects, estimated jointly from measurements of the electric vector spatial coherence function of the incident radiation field measured on each interferometer baseline (Thompson, Moran, \& Swenson 2001, and references therein). The coherence data are sparsely sampled, leading to an ill-posed inverse imaging problem that requires regularization for convergent solution (Cornwell, Braun, \& Briggs 1999). This regularization is typically imposed as a constraint on the properties of the source brightness distributions during deconvolution, including positivity and compact support (Högbom 1974), or via information or entropy measures (Naravan \& Nitvananda 1984; Cornwell \& Evans 1985).

The fidelity of the resulting source brightness distribution cannot be readily estimated given the analytic intractability of the coupled, non-linear calibration and imaging equation, combined with the fact that the parent probability distribution of the measured spatial coherence function is parametrized by the source brightness distribution and the instrumental array calibration, both unknown a prior at the time of observation. Instead, achieved image quality is typically estimated heuristically (Ekers 1986) or by approximate global measures. Common metrics include the ratio of the image brightness root-mean-square (rms) measured in regions of low brightness, $\sigma_{o f f}$, to the thermal noise limit, $\sigma_{t h}$, as calculated for the array from the known antenna sensitivities and receiver and system thermal noise levels, assuming idealized observations of an unresolved point source with perfect array calibration (Wrobel \& Walker 1999). Other measures include the achieved dynamic range $d_{r}$ (which is not a measure of image fidelity per se), expressed as the ratio of the peak brightness, $I_{\text {peak }}$ to the off-source rms, $\frac{I_{\text {peak }}}{\sigma_{\text {off }}}($ Perley 1986), or the use of the deepest negative in a total intensity image (where Stokes $I>0$ ) to derive a scaling relation to reduce the typical under-estimation of $\sigma_{\text {off }}$ Kemball 1993).

These gross measures of image quality are however compromised by their idealized 
underlying assumptions, including that of implied direction-independence of image fidelity across the field. In practice image fidelity is not constant across the field. It is directiondependent (equivalently, pixel-dependent) due to residual, unmodeled instrumental calibration errors jointly or separately in the visibility- or image-plane, and the interaction of these residual errors with non-linear deconvolution effects, amongst other factors. As a result, the assessment of pixel-dependent image fidelity is a general problem in radio interferometry. It is not confined to the important problem of calibrating direction-dependent instrumental errors specifically, as described for example by Bhatnagar et al. (2008).

Although a challenging and largely unsolved problem, understanding pixel-level radiointerferometric imaging fidelity is essential for current and future telescope arrays. Current instruments need to be made more accessible to the larger astronomical community; in particular their user base needs to expand beyond those who have invested the substantial time and effort required to acquire expertise in effective radio-interferometric calibration and imaging data reduction processes (heuristics as summarized for example by Perley, Schwab, \& Bridle (1989)). This is best achieved through the provision of automated pipeline reduction systems; these require associated estimates of image fidelity to allow effective, broad-based community scientific interpretation and analysis.

For leading-edge future arrays, such as the Square Kilometer Array (SKA 1 ), solving the problem of quantitative pixel-level image fidelity assessment is key to their effective design. Any interferometer that cannot reach its target thermal noise limit in a representative integration needed to meet its key science goals is dynamic-range limited. This is important for many interferometers but is particularly acute for the SKA given its high sensitivity. As a result, the SKA has stringent imaging dynamic range requirements (Schilizzi et al. 2007), particularly in continuum observing modes where $d_{r} \sim 10^{6}$ will be required routinely across wide image fields in rapid survey modes and $d_{r} \sim 10^{7}$ for individual, targeted fields. This dynamic range is not achieved routinely by contemporary radio-interferometric arrays except for the innermost pixels in a handful of images, and then only after extensive custom reduction by the most skilled radio interferometry practitioners. With increasing projected radial distance from the field center the dynamic range may typically decline by several orders of magnitude, for reasons noted above. Contemporary examples of high dynamic-range and high-sensitivity observations in radio interferometry, and their associated challenges, are provided by Geller et al. (2000), de Bruvn \& Brentiens (2005), and Norris et al. (2005).

Exponential advances in currently available and anticipated high-performance computing (HPC) capabilities and resources (Bader 2008) allow fundamentally new approaches

\footnotetext{
${ }^{1}$ http://www.skatelescope.org
} 
to the problem of pixel-level radio-interferometric imaging fidelity assessment. Two complementary approaches have recently been reported: a frequentist statistical resampling method (Kemball \& Martinsek 2005, Paper I) and a Bayesian imaging technique (Sutton \& Wandelt 2006). In Paper I, we described the first application of statistical bootstrap resampling techniques to the problem of interferometric imaging fidelity assessment. Statistical resampling is an active area in contemporary statistics research (Efron 2003; Davison. Hinklev, \& Young 2003), and general reviews are provided in recent monographs by Davison \& Hinklev (1997), Chernick (1999), Politis, Romano, \& Wolf (1999), Lahiri (2003), and Zoubir \& Iskander (2004).

In the context of future interferometer arrays, such as the SKA discussed above, the statistical fidelity assessment methods discussed here are particularly important in understanding the optimal design of these telescopes and the contributing factors to their dynamic-range imaging performance. Specifically, although resampling methods may prove too computationally expensive for real-time calibration and imaging at a telescope as computationally demanding as the SKA, they are very valuable tools during SKA design and development. These methods can also be used in off-line analysis of data from interferometer arrays with smaller numbers of elements and lower associated computational costs for calibration and imaging.

We note that resampling techniques as described here are not strongly sensitive to the specific calibration and imaging algorithm in use; their goal is instead to provide a measure of the statistical properties of the underlying calibration and imaging estimator. Our initial evaluation in Paper I considered fidelity assessment of polarization calibration for Very Long Baseline Interferometry (VLBI) arrays, a representative radio-interferometric calibration and imaging problem of interest in its own right (Kemball 1999). The statistical performance of both model-based and subsample bootstrap resampling was evaluated by inter-comparison of the bootstrap results with those obtained by direct Monte Carlo simulation for a single fixed two-component polarized source model and array configuration. Our initial study found both bootstrap resampling techniques to be computationally tractable with modern HPC resources and to have good statistical performance for image variance estimation for the single source model and array configuration considered in that initial study.

In the current paper we describe results from broader evaluation of the applicability of statistical resampling to radio-interferometric imaging fidelity assessment. We extend the scope of our original evaluation in Paper I by expanding the problem domain along two axes, namely source model structure and array parallactic angle coverage. Both parameters vary the expected degree of systematic error in the polarimetric calibration and imaging estimator used in the study and therefore the pixel-level morphology and magnitude of the 
image fidelity distribution over which the bootstrap resampling methods can be evaluated.

In the expanded evaluation reported here, we find here that the model-based and subsample bootstrap resampling methods for data with long-range statistical dependence, used in Paper I, continue to show good statistical performance when applied over a wider range of observing configurations and source models. We refine guidelines and rules for their general use in radio interferometry and report initial results on their applicability to image bias estimation.

The paper is structured as follows. In Section 2 the theory of bootstrap resampling is summarized, as applied to the interferometric calibration and imaging problem under examination. Section 3 describes the numerical simulation methods used to measure the statistical performance of the bootstrap resampling techniques under evaluation. Simulation results are presented in Section 4 and discussed in Section 5. In Section 6, we summarize the conclusions from the current work.

\section{Theory}

In this section, we recap the key elements of the statistical resampling method used in the current study for fidelity assessment of interferometric polarization calibration and imaging. A full description of the theory can be found in Paper I.

\subsection{The imaging equation for radio interferometry}

We adopt the radio-interferometric imaging equation developed by Hamaker. Bregman. \& Sault (1996), Sault, Hamaker, \& Bregman (1996), Hamaker \& Bregman (1996) and Hamaker (2000), and generalized for image-plane effects by Cornwell (1995) and Noordam (1995):

$$
V_{m n}=\prod_{\kappa}\left[G_{m}^{\kappa} \otimes G_{n}^{\kappa *}\right] \int_{\Omega} \prod_{\kappa}\left[T_{m}^{\kappa}(\vec{\rho}) \otimes T_{n}^{\kappa *}(\vec{\rho})\right] e^{-2 \pi j \vec{b}_{m n} \cdot\left(\vec{\rho}-\overrightarrow{\rho_{s}}\right)} K S(\vec{\rho}) d \Omega
$$

where $j=\sqrt{-1}$, the term $V_{m n}$ is the measured complex 4-vector of polarization correlations on the baseline $\vec{b}_{m n}$ between antennas $m$ and $n$ (by common convention referred to as visibilities in this discipline), $S(\vec{\rho})$ is the Stokes 4 -vector of radio brightness in unit direction $\vec{\rho}$, vector $\overrightarrow{\rho_{s}}$ is the center of the field $\Omega$, and $K$ is a constant $(4 \times 4)$ matrix that maps the Stokes parameters $\{I, Q, U, V\}$ into the polarization receptor basis (e.g. orthogonal circular or linear) of the visibility polarization correlations. Direction-independent gains 
of instrumental type $\kappa$ at antenna $m$ are denoted $G_{m}^{\kappa}$, with associated direction-dependent gains $T_{m}^{\kappa}=f(\vec{\rho})$; both are $(2 \times 2)$ Jones matrices in the polarization receptor basis, and of arbitrary (e.g. pixel or functional) parametrization. The outer matrix product is denoted by $\otimes$, and complex conjugation by an asterisk.

\subsection{Polarization self-calibration}

For the interferometric polarization self-calibration and imaging problem considered here as a representative problem for the study of imaging fidelity assessment, the imaging equation 1 contains only direction-independent Jones matrices $G_{m}^{\kappa}=\left\{P_{m}, D_{m}\right\}$, i.e. $T_{m}^{\kappa}$ is the null set $\{\emptyset\}$ (or equivalently, the $T_{m}^{\kappa}$ are identity matrices), and where $P_{m}=$ $\operatorname{diag}\left(\mathrm{e}^{-\mathrm{j} \alpha_{\mathrm{m}}(\mathrm{t})}, \mathrm{e}^{+\mathrm{j} \alpha_{\mathrm{m}}(\mathrm{t})}\right)$ is the Jones matrix containing the feed parallactic angle term $\alpha_{m}(t)$, known analytically, and $D_{m}$ is an anti-diagonal matrix containing the antenna-based instrumental polarization leakage terms for each nominally orthogonal polarization receptor basis. As described in Paper I, our polarization self-calibration method, as a joint iterative solution for $D_{m}$ and $S(\vec{\rho})$, is a subset of general self-calibration within the imaging equation framework. For each cycle of (non-progressive) iterative refinement of $S(\vec{\rho})$, we solve for the instrumental polarization by minimizing $\chi^{2}$, formed as the $L_{2}$ complex norm at the position of the unknown $D_{m}$ in equation 1, by integrating the imaging equation from both the right-hand and left-hand sides to that position.

\section{3. $\quad$ Imaging fidelity assessment by bootstrap resampling}

Before considering bootstrap resampling as a technique for imaging fidelity assessment we need to formulate the problem of radio-interferometric calibration and imaging as a statistical inference problem. General statistical inference and estimation in signal processing is described by Kay (1993). The measured visibility dataset, $V_{m n}^{o b s}=V_{m n}+\mathcal{N}, \forall(m, n, t)$, can be considered a single realization of a vector over time $t$ of random variables $\mathcal{V}$ drawn from a joint, multi-variate parent probability density function (PDF) of parametric form $p\left(\mathcal{V} ; S(\vec{\rho}), D_{m}, \sigma_{t h}\right)$. Independent, identically-distributed (IID) thermal noise in each visibility measurement is denoted by $\mathcal{N}$. We consider the joint self-calibration solvers for $D_{m}$ and $S(\vec{\rho})$ as statistical point estimators, denoted $\hat{D}_{m}$ and $\hat{S}(\vec{\rho})$ respectively. In this standard statistical inference framework, the problem of imaging fidelity assessment is that of determining the sampling distribution $\mathcal{F}_{\hat{S}(\vec{\rho})}$ of $\hat{S}(\vec{\rho})$. As described in Paper I, this problem is neither tractable analytically, nor is $p\left(\mathcal{V} ; S(\vec{\rho}), D_{m}, \sigma_{t h}\right)$ (or therefore $\mathcal{F}_{\hat{S}(\vec{\rho})}$ ) known a priori. 
Statistical resampling offers an alternative inference method for $\mathcal{F}_{\hat{S}(\vec{\rho})}$ that does not require analytic tractability or detailed knowledge of $p\left(\mathcal{V} ; S(\vec{\rho}), D_{m}, \sigma_{t h}\right)$. These methods are computationally expensive however, but contemporary advances in HPC capabilities now make them viable approaches. The single realization of $\mathcal{V}$, comprising the observed visibility dataset, can be used to construct an empirical distribution function $\tilde{p}\left(\mathcal{V} ; S(\vec{\rho}), D_{m}, \sigma_{t h}\right)$. Resamples $\mathcal{V}^{*}$ drawn from $\tilde{p}\left(\mathcal{V} ; S(\vec{\rho}), D_{m}, \sigma_{t h}\right)$, conditional on the observed data $V_{m n}^{\text {obs }}$, and under statistical conditions where the bootstrap is applicable, mirror the statistical relationship between $\mathcal{V}$ and the unknown parent distribution $p\left(\mathcal{V} ; S(\vec{\rho}), D_{m}, \sigma_{t h}\right)$. The imaging estimator $\hat{S}(\vec{\rho})$, is here chosen to represent the estimator for the restored image. Acting on the resampled visibility datasets, this estimator yields images $S^{*}$; their statistical distribution relative to $S_{x y_{0}}^{*}$, which is obtained from the $\hat{S}(\vec{\rho})$ acting on the observed (template) realization, provides a bootstrap estimate of $\mathcal{F}_{\hat{S}(\vec{\rho})}$ and hence an assessment of imaging fidelity by our current definition. Here the subscript $x y$ denotes the use of a pixel basis for the images.

Radio-interferometric data have long-range statistical dependence and thus require bootstrap innovations developed for dependent data (Lahiri 2003). In Paper I, we demonstrated the successful use of two such methods, namely the model-based and subsample bootstraps, for a single simulated test case in polarization self-calibration and imaging. We present an expanded evaluation in the following sections.

\section{Simulation methods}

\subsection{Run codes}

The primary goal of this study is to evaluate the statistical performance of the bootstrap resampling techniques advanced in Paper I over a larger domain subset. We expand the previous study along two axes, namely the expected level of image fidelity and the range of source brightness distribution morphologies considered. We retain the same interferometric polarization and imaging problem used in Paper I as a representative test problem in this discipline. The details of our polarization self-calibration heuristic is described in detail in Paper I; broadly summarized, this is a joint solver for $S(\vec{\rho})$ and $D_{m}$ over ten non-progressive self-calibration iterations, starting from an unpolarized unit-brightness point source brightness distribution and zero instrumental polarization for each antenna in the array. In the 
current work, we also retain the the simulated Very Long Baseline Array (VLBA2) configuration and instrumental polarization terms enumerated in Table 1 of Paper I.

To vary the expected image fidelity, we adjust the range of parallactic angle for the simulated array configuration by truncating the simulated observation duration. The VLBA is comprised of antennas with azimuth-elevation antenna mounts which, unlike equatorial mounts, produce non-zero, time-variable feed parallactic angle variations (Thompson. Moran, \& Swenson 2001). These parallactic angle ranges are labeled as run codes A through F, in order of decreasing parallactic angle range, and are enumerated in Table 1. A graphical representation is provided in Figure 1. Run code $\mathrm{A}$ is the case of nearly-complete parallactic angle coverage considered as the sole case studied in Paper I. Interferometric polarization calibration of linearly-polarized calibrators has a higher degree of systematic error for reduced parallactic angle coverage; this results from the increasing degeneracy introduced between the polarization basis functions $e^{ \pm j \alpha_{m}(t)}$ and the unknown full-polarization source brightness distribution $S(\vec{\rho})$ for low parallactic angle range (Morris, Radhakrishnan, \& Seielstad 1964; Conway \& Kronberg 1969; Cotton et al. 1984; Roberts et al. 1984).

To vary source polarization morphology, we decrease the angular separation between the two simulated Gaussian components used in Paper I (as parametrized in Table 2 of Paper I), so increasing their degree of spatial overlap and hence polarization complexity. We reproduce the source model in Stokes $\{I, Q, U, V\}$ at the original component separation used in Paper I in Figure 2, and include the quantitative component parameter values in the caption to that Figure. The component separations used in the current study of source morphology variation are tabulated in Table 2 in this paper as run codes X, Y, and Z. For these run codes, however, we retain the same complete parallactic angle range used in Paper I, i.e. the same parallactic angle range as run code $\mathrm{A}$ in Table 1 .

\subsection{Monte Carlo reference simulations}

For each run code $\{\mathrm{A}-\mathrm{F}, \mathrm{X}-\mathrm{Z}\}$, we assess the statistical performance of each bootstrap resampling method by inter-comparison with results obtained by direct Monte Carlo simulation. As in Paper I, an ensemble of $N_{s}=256$ visibility datasets were generated for each run code by direct Monte Carlo simulation. Additive IID thermal noise contributions $\mathcal{N}$ were drawn as a phasor from the complex normal distribution $\mathcal{C N}\left(0, \sigma_{t h}^{2}\right)$; this distribution is defined by Kav (1993). The joint polarization self-calibration and imaging estimator for $\hat{D}_{m}$

\footnotetext{
${ }^{2}$ The National Radio Astronomy Observatory is a facility of the National Science Foundation operated under agreement by Associated Universities, Inc.
} 
and $\hat{S}(\vec{\rho})$, was applied to each visibility dataset realization within the Monte Carlo ensemble generated for each run code. The bias and mean-squared error (MSE) of the sampling distribution $\mathcal{F}_{\hat{S}(\vec{\rho})}$ of $\hat{S}(\vec{\rho})$ were then computed for each restored ensemble image, in a pixel basis $S_{x y}$, relative to the true source model brightness distribution $\breve{S_{x} y}$ used to generate the simulated data, the latter convolved with a common median restoring beam matched separately to the spatial resolution of each run code. The variance was computed as $N_{s}^{-1} \sum\left(S_{x y}^{2}\right)-{\overline{S_{x y}}}^{2}$, where $\overline{S_{x y}}$ is the sample mean across the ensemble, and the MSE per pixel relative to the true model was computed as $N_{s}^{-1} \sum\left(S_{x y}-\widetilde{S_{x} y}\right)^{2}$. Each statistic was computed per image pixel, per self-calibration iteration number, and per Stokes parameter $\{I, Q, U, V\}$. These Monte Carlo estimates of the variance, bias, mean, and MSE of the sampling distribution $\mathcal{F}_{\hat{S}(\vec{\rho})}$ of the imaging estimator $\hat{S}(\vec{\rho})$ provide an estimate of truth against which the statistical performance of the bootstrap resampling methods can be assessed.

\subsection{Bootstrap simulations}

Both the model-based and subsample bootstrap methods described in Paper I were used in the current study. These bootstrap methods are applicable to data with longterm statistical dependence, as is the case in radio interferometry. The parameters for each bootstrap method, both the model-based (M1-M4) and subsample (S1-S4) bootstrap are reproduced here from Paper I as Table 3 and Table 4 respectively. The reader is referred to Paper I for specific implementation details for these bootstrap methods. For each run code $\{\mathrm{A}-\mathrm{F}, \mathrm{X}-\mathrm{Z}\}$, a bootstrap ensemble (of size 256 realizations) was generated for each separate bootstrap method $\{\mathrm{M} 1-\mathrm{M} 4, \mathrm{~S} 1-\mathrm{S} 4\}$ by resampling from a single template realization (here chosen to be either the 127th or 128th of $N_{s}=256$ ) taken from the matching Monte Carlo ensemble for that run code. For each resulting bootstrap ensemble, the bias and MSE of the sampling distribution of the imaging estimator, $\hat{S}(\vec{\rho})$, were computed from the restored images obtained across the ensemble, $S_{x y}^{*}$, relative to the restored image, $S_{x y_{0}}^{*}$, obtained for the template visibility dataset realization. This technique can be used directly with real data obtained by physical observation, unlike Monte Carlo simulation, which presupposes knowledge of the unknown true source model.

The primary purpose of this study is to assess if a single observed visibility dataset realization can be used, through resampling, to estimate imaging fidelity, quantified here as the variance, bias, and MSE of the sampling distribution $\mathcal{F}_{\hat{S}(\vec{\rho})}$ of a regularized imaging estimator $\hat{S}(\vec{\rho})$. We assess the statistical performance of each bootstrap method in the only practical way, namely against the sampling distribution properties obtained by direct Monte Carlo simulation. Conclusions regarding the statistical applicability of bootstrap 
resampling for imaging fidelity obtained in this way translate directly to real observations if the general validity of the approach can be assessed numerically over a sufficiently complete domain subset. As part of that more complete evaluation, for each run code $\{\mathrm{A}-\mathrm{F}, \mathrm{X}-\mathrm{Z}\}$, we have completed a Monte Carlo simulation (denoted by code MC) and a set of resampling simulations for bootstrap codes $\{\mathrm{M} 1-\mathrm{M} 4, \mathrm{~S} 1-\mathrm{S} 4\}$.

\subsection{HPC implementation}

As in Paper I, a modified version of the AIPS++3 package was used to perform polarization self-calibration and imaging, but for the work described in this paper, compiled within the build framework for radio-interferometric analysis software described by Kemball, Crutcher, \& Hasan (2008). In addition to the changes to the code base to support polarization self-calibration and statistical resampling, we also replaced the default $\chi^{2}$ Newton solver for the imaging equation in the original package with a conjugate-gradient solver as implemented in the OptSolve ++ packag 4 .

The computations were parallelized over visibility dataset realization within each bootstrap or Monte Carlo ensemble and run on a 32-bit Intel Xeon Linux cluster operated as a national computational resource by the National Center for Supercomputing Application 5 . The cluster has a peak single-precision floating point performance per processor of approximately 12 Gflops. Each run was typically distributed over 64 processors and ran for $\sim 3-4$ hours resulting in an integrated compute cost of $O$ (Tflops). We note as before, however, that the problem is highly parallelizable and scalable over realization and has good load balancing characteristics; only the template realization and final statistical processing need to be performed in serial segments of the code.

\section{Simulation results}

The performance of the polarization self-calibration algorithm in direct Monte Carlo simulations over run codes $\{\mathrm{A}-\mathrm{F}\}$ in shown in Figure 3 , these run codes have parallactic angle ranges depicted in Figure 1. Here the MSE of the instrumental polarization estimator $\hat{D}_{m}$, averaged over antenna and receptor polarization, with reference to the known truth used

\footnotetext{
${ }^{3}$ http://aips2.nrao.edu

${ }^{4}$ OptSolve ++ is distributed by Tech-X corporation (http://www.techxhome.com)

${ }^{5}$ www.ncsa.uiuc.edu
} 
in the simulations, is separately plotted for each run code against self-calibration iteration number. The detailed computation of the MSE for $\hat{D}_{m}$ is described in the caption to Figure 3 ,

As described in preceding sections, the primary purpose of the current work is to assess the statistical performance of the bootstrap resampling techniques advanced in Paper I, over an expanded domain subset in this discipline. This has been achieved by increasing the range of imaging fidelity and source morphologies considered, enumerated by run codes $\{\mathrm{A}-$ F, X-Z \} in Tables 1 and 2, For each run code, a direct Monte Carlo simulation (MC) was performed and bootstrap resampling methods $\{$ M1-M4, S1-S4\} subsequently applied to a single visibility dataset drawn from each Monte Carlo ensemble.

For a qualitative assessment of the statistical performance of the bootstrap methods relative to the Monte Carlo reference, we plot in Figures 4 through 7 the pixel-based Stokes $Q$ variance images for the final iteration of polarization self-calibration for a subset of run codes, chosen here to be $\{\mathrm{B}, \mathrm{D}, \mathrm{X}, \mathrm{Z}\}$; these run codes are representative of typical bootstrap statistical performance observed. We choose Stokes $Q$ here as both Stokes $\{Q, U\}$ show residual calibration errors most clearly for the underlying polarization calibration algorithm. In each of these figures, the reference Monte Carlo variance image is shown in the upper left corner; the remaining images, in sequential order, are the variance images obtained by bootstrap resampling methods $\{\mathrm{M} 1-\mathrm{M} 4, \mathrm{~S} 1-\mathrm{S} 4\}$. A default color mapping was used in generating the raster images in these figures; for each image the same color palette was mapped to the range of pixel data values in the image variance cube resulting from each run; each cube has axes $(x, y)$, Stokes parameter $\{I, Q, U, V\}$, and self-calibration iteration number. The variance images contain contributions from both calibration and deconvolution errors, as discussed in the introduction.

For a quantitative assessment of bootstrap statistical performance we use a similar goodness-of-fit statistic $v_{M S E}$, to that defined in Paper I to compare the variance image obtained using a given bootstrap resampling method, $v a r_{x y}$, to the the reference variance image obtained by Monte Carlo simulation, $\operatorname{var}_{x y}^{M C}$. In the current work, the statistic is computed over the inner quarter of the image, all Stokes parameters $\{I, Q, U, V\}$, and all self-calibration iteration numbers $k=1-10$, as:

$$
v_{M S E}=\frac{1}{N} \sum_{I Q U V} \sum_{j=1}^{10} \sum_{\Omega_{\frac{1}{4}}}\left(\frac{v^{a} r_{x y}}{v_{f}}-v a r_{x y}^{M C}\right)^{2}
$$

were $v_{f}$ is the variance scaling factor (Davison \& Hinkley 1997).

The value of $v_{f}$ was determined numerically for each bootstrap run in a Newton mini- 
mization of the goodness-of-fit statistic $v_{M S E}$, as defined in equation 2. The resulting fitted values of $v_{f}$ are listed in Table 5, and the associated minima in $v_{M S E}$ given in Table 6, both cross-tabulated by run code $\{\mathrm{A}-\mathrm{F}, \mathrm{X}-\mathrm{Z}\}$ and bootstrap code $\{\mathrm{M} 1-\mathrm{M} 4, \mathrm{~S} 1-\mathrm{S} 4\}$. The values of $v_{M S E}$ in Table 6 are normalized per row by the minimum value obtained across bootstrap codes $\{\mathrm{M} 1-\mathrm{M} 4, \mathrm{~S} 1-\mathrm{S} 4\}$ for each run code $\{\mathrm{A}-\mathrm{F}, \mathrm{X}-\mathrm{Z}\}$. The minimum $v_{M S E}$ is given in the right-most column of the table. The optimal bootstrap methods are therefore indicated in this table by unit relative $v_{M S E}$. Note that the values of $v_{M S E}$ given in Table 5 of Paper I were incorrectly scaled in absolute magnitude and need to be multiplied by a factor $1.502 \times 10^{-5}$ for comparison with the $v_{M S E}$ values in the current work.

In Figures 8 through 10 we plot the pixel-based Stokes $Q$ variance for the final polarization self-calibration iteration for each run code $\{\mathrm{A}-\mathrm{F}, \mathrm{X}-\mathrm{Z}\}$ for both the direct Monte Carlo reference simulation and the optimal model-based and subsample bootstrap methods identified in Table 6. In these figures, the Stokes $Q$ variance images are drawn as contour plots with identical fractional contour levels in each figure sub-panel.

\section{Discussion}

The results presented in the previous section show that the applicability of statistical resampling as a tool for imaging fidelity assessment in radio interferometry is upheld from our initial evaluation presented in Paper I, when explored over a larger domain subset, here a greater range of expected image fidelity and source brightness distribution morphology. The current work continues to support the conclusion that contemporary statistical resampling techniques developed for data with long-range statistical dependence (Lahiri 2003) are a promising method for estimating the statistical properties of regularized calibration and imaging estimators used for the solution of the imaging equation 1 in radio interferometry. The technique also intrinsically allows an estimate of image quality per pixel, in contrast to coarser direction-independent metrics, such as those based on off-source rms $\sigma_{o f f}$ for example. As a bootstrap technique, it is also not sensitive to assumptions about the functional form or parametrization of the parent PDF of the measured visibility data, and does not require the frequent idealization of uniform Gaussianity for the total noise contribution.

As described in the preceding section, we assess each bootstrap method quantitively against the direct Monte Carlo variance reference image; this goodness-of-fit statistic $v_{M S E}$ is tabulated in Table 6. An optimal bootstrap method, within a given set, is the method that agrees most closely with the Monte Carlo result by this quantitative measure.

Our finding in Paper I that the model-based bootstrap generally strongly outperforms 
the subsample bootstrap continues to hold true in the broader evaluation described here. The statistical performance of the subsample bootstrap improves with increasing subsample delete fraction $f_{s}$, as expected theoretically (Davison \& Hinkley 1997). The subsample bootstrap performance drops precipitously for low or moderate delete fraction $f_{s}<0.5$ and the optimal subsample bootstrap code is found to be $\mathrm{S} 4$ consistently across all run codes $\{\mathrm{A}-\mathrm{F}, \mathrm{X}-\mathrm{Z}\}$ in the current study. The subsample delete fraction for bootstrap code S4 is 0.75 , the highest value of $f_{s}$ amongst the bootstrap codes $\{\mathrm{S} 1-\mathrm{S} 4\}$. Despite the poorer statistical performance of the subsample bootstrap, we note that it has the advantage of ease of implementation and requires no free user-adjustable parameters if a fixed value $f_{s}=0.75$ is adopted.

The optimal model-based bootstrap code is found to be M3 across run codes $\{\mathrm{A}-\mathrm{F}\}$ and M4 for run codes $\{\mathrm{X}-\mathrm{Z}\}$. The best single bootstrap over all run codes is M3. The model-based bootstrap used in this study models the long-term statistical dependence in the data by fitting a series of independent segmented polynomial fits (of maximum degree $N_{p}$ ) to the visibility time-series on each interferometer baseline over successive solution intervals of length $\Delta t_{b}$. This does not require a priori knowledge of the source brightness distribution, however. Full details of the implementation of this method can be found in Paper I. Bootstrap codes M3 and M4 have increasing solution intervals $\Delta t_{b}^{M 4}>\Delta t_{b}^{M 3}$ (as enumerated in Table 3 ). The optimality of M4 over M3 for run codes $\{\mathrm{X}-\mathrm{Z}\}$ is consistent with the more compact source morphology for these run codes, as noted in Table 2, and therefore a lower mean rate of change of source visibility over time. As shown in Table 6, the relative performance of the model-based bootstrap shows a weak dependence on $\Delta t_{b}$, compared to the strong dependence of the subsample bootstrap performance on the delete fraction $f_{s}$. In this sense the modelbased bootstrap is also more robust against sub-optimal bootstrap parameter choices. The current study confirms that $\Delta t_{b}$ should be chosen with regard to the expected mean time rate of change of the visibility function. This can also in principle be deduced automatically from the actual observed time-variability in $V_{m n}^{o b s}$. The interval $\Delta t_{b}$ needs to be chosen as the longest interval over which the piece-wise polynomial model for the statistical dependence in the visibility time-series holds on each interferometer baseline.

For the subsample bootstrap, the variance scaling factor $v_{f}$ is expected to have a powerlaw dependence on subsample delete fraction $f_{s}$. Davison \& Hinkley (1997) quote an expected relation $v_{f} \sim\left(1-f_{s}\right)^{-a}$ for the related delete-d jacknife method. The fitted scaling factors from the run codes $\{\mathrm{A}-\mathrm{F}, \mathrm{X}-\mathrm{Z}\}$ in the current study are plotted against subsample delete fraction $f_{s}$ in Figure 12. We find the relation to be broadly consistent across the different parallactic angle ranges, which have different numbers of visibilities $N_{v i s}$, and the source morphologies considered in the current work. We find empirically in the current study that a function of the form $v_{f}=b e^{-a\left(1-f_{s}\right)}$ provides a better fit to the data (either separately 
for each run code or jointly across all run codes) than $v_{f}=b\left(1-f_{s}\right)^{a}$ or $v_{f}=a f_{s}^{2}+b f_{s}+c$, although we stress that the current data do not statistically rule out these models. Further studies with a finer sampling in $f_{s}$ are planned to constrain this relation more closely. Additional work will also be required to determine its broader applicability; this is an empirical relation in its current form. A joint best fit across run code $\{\mathrm{A}-\mathrm{F}, \mathrm{X}-\mathrm{Z}\}$ is drawn as a solid line in Figure 12 in the form:

$$
v_{f}\left(f_{s}\right)=4.481 e^{-3.287\left(1-f_{s}\right)}
$$

The subset of runs comprising the direct Monte Carlo simulations over parallactic angle ranges $\{\mathrm{A}-\mathrm{F}\}$ provide an opportunity to study the performance of the polarization selfcalibration algorithm introduced in Paper I. The total MSE for the estimator $\hat{D}_{m}$, averaged over antenna and polarization receptor basis, is plotted on a logarithmic scale against selfcalibration iteration number in Figure 3. As noted, and demonstrated, in Paper I, the algorithm is expected, from relative information arguments, to demonstrate rapid convergence for the case of good parallactic angle coverage. The current study measures the algorithm performance for decreasing parallactic angle range over run codes $\{\mathrm{A}-\mathrm{F}\}$. Figure 3 shows that there is good convergence for parallactic angle ranges $\Delta \alpha \sim 80-140^{\circ}$ that include source transit, moderate convergence for $\Delta \alpha \sim 30-60^{\circ}$, and poor convergence for $\Delta \alpha \sim 0^{\circ}$. This is consistent with heuristic rules of thumb for other interferometric polarization calibration techniques that recommend a minimum parallactic angle range $\Delta \alpha>100^{\circ}$ (Leppanen, Zensus, \& Diamond 1995).

Although the current study has focused on measuring the variance of the sampling distribution of the imaging estimator $\hat{S}(\vec{\rho})$, we show initial results on bias estimation in Figure 11, These example images are selected for their expected high levels of bias, as anticipated in the current study for the case of Stokes $\{Q, U\}$ images at low polarization self-calibration iteration numbers, where the instrumental polarization terms will still have high MSE. As for the variance study, we assess the statistical performance of the bootstrap resampling estimate of bias against the result obtained by direct Monte Carlo simulation. Figure 11 shows that the optimal variance estimators, as identified in Table 6, show qualified promise as bias estimators in the high-bias regime. However, we note that our preliminary experience is that they have lower statistical performance than variance estimators. Efron \& Tibshirani (1993) discuss the performance of bootstrap bias estimators, and propose techniques that can significantly improve their statistical performance. We intend to examine bias estimation in this application in more detail in a future paper.

We also note that new statistical imaging techniques of the type considered in this paper allow novel approaches to understanding direction-dependent radio-interferometric 
calibration and imaging fidelity, especially those challenges posed by wide-field imaging at uniform, high dynamic range, as needed for future leading-edge telescopes such as the SKA. These techniques offer a new approach, distinct from instrument simulation which has wellknown limitations, to derive direction-dependent imaging fidelity assessments from single observations with real prototype arrays.

The computational cost of the bootstrap resampling techniques evaluated here is approximately $\sim O\left(10^{2}\right)$ times the processing cost for a single dataset without resampling. This places it well within contemporary HPC resources at the teraflop scale and those projected to be available soon in the petascale era (Bader 2008). The cost of the algorithm is strongly mitigated by its high degree of scalability and effective parallel decomposition.

\section{Conclusions}

We draw the following conclusions from the current evaluation of bootstrap resampling for radio-interferometric imaging fidelity assessment over a broader domain subset:

1. Statistical resampling techniques for dependent data remain a promising approach to the important problem of pixel-level fidelity assessment in radio-interferometric calibration and imaging. They show good statistical performance as assessed against direct Monte Carlo simulation, and are computationally affordable and scalable on contemporary and future HPC resources. They have the desirable statistical properties of independence on the detailed functional form or parametrization of the parent PDF of the observed visibility data and of not requiring an analytic solution for the calibration and imaging inference problem.

2. Model-based bootstrap techniques outperform subsampling bootstrap methods in general in our study, although both are of value in this application. The subsample bootstrap performs well for delete fraction $f_{s} \sim 0.75$, is straight-forward to implement, and has no adjustable parameters if $f_{s}$ is adopted as this value. The model-based bootstrap has superior statistical performance in general. It has free parameters $\left(N_{p}, \Delta t_{b}\right)$ that can in principle be deduced from the observed data and against which the method is relatively robust to the exact values used.

3. The subsample bootstrap variance scaling relation $v_{f}=f\left(f_{s}\right)$ measured in the current study shows relatively limited variability across the broader domain subset considered here. The current data are consistent with an exponential function: $v_{f}=b e^{-a\left(1-f_{s}\right)}$. 
4. The polarization self-calibration algorithm introduced in Paper I is found to converge at a faster than exponential rate for good parallactic coverage $\Delta \alpha$. Its performance for lower parallactic angle ranges is found to deteriorate for $\Delta \alpha<100^{\circ}$ and to break down in the limit as $\Delta \alpha \rightarrow 0$ as expected.

5. An initial examination of statistical resampling for bias estimation shows qualified promise in the high-bias regime in this discipline, but further evaluation is required.

This material is based upon work supported by the National Science Foundation under Grant Nos. AST-0506745 and AST-0431486. Any opinions, findings, and conclusions or recommendations expressed in this material are those of the authors and do not necessarily reflect the views of the National Science Foundation. This work was also partially supported by the National Center for Supercomputing Applications under AST-050003 and used the system tungsten.ncsa.uiuc.edu.

\section{REFERENCES}

Bader, D.A. (ed.) 2008, Petascale Computing: Algorithms and Applications (Boca Raton, FL: Chapman \& Hall/CRC)

Bhatnagar, S., Cornwell, T.J., Golap, K., \& Uson, J.M. 2008, A\&A, 487, 419

Chernick, M.R. 1999, Bootstrap Methods: A Practitioner's Guide (New York: Wiley)

Conway, R.G., \& Kronberg, P.P. 1969, MNRAS, 142, 11

Cornwell, T.J., Braun, R., \& Briggs, D.S. 1999, in ASP Conf. Ser. 180, Synthesis Imaging in Radio Astronomy II, A Collection of Lectures from the Sixth NRAO/NMIMT Synthesis Imaging Summer School, ed. by G.B. Taylor, C.L. Carilli, \& R. A. Perley (San Francisco:ASP), 151

Cornwell, T.J. \& Evans, K.F. 1985, A\&A, 143, 77

Cornwell, T.J. 1995, The Generic Interferometer I: Overview of Calibration and Imaging, AIPS++ Implementation Note 183, http://aips2.nrao.edu/docs/notes/183/183.html

Cotton, W.D., Geldzahler, B.J., Marcaide, J.M., Shapiro, I.I., Sanroma, M., \& Rius, A. 1984, ApJ, 286, 503 
Davison, A.C., \& Hinkley, D.V. 1997, Bootstrap Methods and their Applications (Cambridge: CUP)

Davison, A.C., Hinkley, D.V., \& Young, G.A. 2003, Stat.Sci., 18, 141

de Bruyn, A.G. \& Brentjens, M.A. 2005, A\&A, 441, 931

Efron, B. \& Tibshirani, R. 1993, An Introduction to the Bootstrap (New York, NY:Chapman \& Hall)

Efron, B. 2003, Stat.Sci., 18, 135

Ekers, R.D. 1986, in Synthesis Imaging, Course Notes from an NRAO Summer School Held in Socorro, New Mexico, August 5-9, 1985, eds. R.A. Perley, F.R. Schwab, \& A.H. Bridle (Green Bank WV:NRAO), 149

Geller, R.M., Sault, R.J., Antonucci, R., Killeen, N.E.B., Ekers, R., Desai, K., \& Whysong, D. 2000, ApJ, 539, 73

Lahiri, S.N. 2003, Resampling Methods for Dependent Data (New York: Springer-Verlag)

Hamaker, J.P., Bregman, J.D., \& Sault, R.J. 1996, A\&AS, 117, 137

Hamaker, J.P., \& Bregman, J.D. 1996, A\&AS, 117, 161

Hamaker, J.P. 2000, A\&AS, 143, 515

Högbom, J.A. 1974, A\&AS, 15, 417

Kay, S.M. 1993, Fundamentals of Statistical Signal Processing: Estimation Theory (New Jersey: Prentice Hall)

Kemball, A.J. 1993, Ph.D. thesis, Rhodes University

Kemball, A.J. 1999, in ASP Conf. Ser. 180, Synthesis Imaging in Radio Astronomy II, A Collection of Lectures from the Sixth NRAO/NMIMT Synthesis Imaging Summer School, ed. by G.B. Taylor, C.L. Carilli, \& R. A. Perley (San Francisco:ASP), 499

Kemball, A. \& Martinsek, A. 2005, AJ, 129, 1760

Kemball, A.J., Crutcher, R.M., \& Hasan, R. 2008, Softw.Pract.Exper., 38, 493

Leppanen, K.J., Zensus, J.A., \& Diamond, P.J. 1995, AJ, 110, 2479

Morris, D., Radhakrishnan, V., \& Seielstad, G.A. 1964, ApJ, 139, 551 
Narayan, R. \& Nityananda, R., in Indirect Imaging, ed. J.A. Roberts (Cambridge:CUP), 281.

Noordam, J.E. 1995, Some Practical Aspects of the Matrix-based Measurement Equation of a Generic Radio Telescope, AIPS++ Implementation Note 182, http://aips2.nrao.edu/docs/notes/182/182.html

Norris, R.P., Huynh, M.T., Jackson, C.A., Boyle, B.J., Ekers, R.D., Mitchell, D.A., Sault, R.J., Wieringa, M.H., Williams, R.E., Hopkins, A.M., \& Higdon, J. 2005, AJ, 130, 1358

Perley, R.A. 1986, in Synthesis Imaging, Course Notes from an NRAO Summer School Held in Socorro, New Mexico, August 5-9, 1985, eds. R.A. Perley, F.R. Schwab, \& A.H. Bridle (Green Bank WV:NRAO), 161

Perley, R.A., Schwab, F.R., \& Bridle, A.H., editors 1989, ASP Conf. Ser. 6, Synthesis Imaging in Radio Astronomy, A Collection of Lectures from the Third NRAO Synthesis Imaging Summer School, ed. by R.A. Perley, F.R. Schwab, \& A.H. Bridle (San Francisco: ASP)

Politis, D.N., Romano, J.P., \& Wolf, M. 1999, Subsampling (New York: Springer-Verlag)

Roberts, D.H., Potash, R.I., Wardle, J.F.C., Rogers, A.E.E., \& Burke, B.F. 1984, in IAU Symp. 110, VLBI and Compact Radio Sources, ed. R. Fanti, K. Kellermann, \& G. Setti (Dordrecht:Reidel), 35

Sault, R.J., Hamaker, J.P., \& Bregman, J.D. 1996, A\&AS, 117, 149

Schilizzi, R.T., Alexander, P., Cordes, J.M., Dewdney, P.E., Ekers, R.D., Faulkner, A.J., Gaensler, B.M., Hall, P.J., Jonas, J.L., \& Kellermann, K.I. 2007, Preliminary Specifications for the Square Kilometer Array (Manchester, UK: SKA Project), http://www.skatelescope.org/PDF/Preliminary_SKA_Specifications.pdf

Sutton, E.C. \& Wandelt, B.D. 2006, ApJS, 162, 401

Thompson, A.R., Moran, J.M., \& Swenson Jr., G.W. 2001, Interferometry and Synthesis in Radio Astronomy (2d ed.; New York: Wiley)

Wrobel, J.M. \& Walker, R.C. 1999, in ASP Conf. Ser. 180, Synthesis Imaging in Radio Astronomy II, A Collection of Lectures from the Sixth NRAO/NMIMT Synthesis Imaging Summer School, ed. by G.B. Taylor, C.L. Carilli, \& R. A. Perley (San Francisco:ASP), 171 
Zoubir, A.M. \& Iskander, D.R. 2004, Bootstrap Techniques for Signal Processing (Cambridge, UK:CUP) 
Table 1. Parallactic angle ranges and run codes

\begin{tabular}{lrrrr}
\hline \hline & $\begin{array}{r}\left|\Delta \alpha_{1}\right| \\
\text { Run code }\end{array}$ & $\begin{array}{c}\left|\Delta \alpha_{2}\right| \\
(\mathrm{deg})\end{array}$ & $\begin{array}{c}|\overline{\Delta \alpha}| \\
(\mathrm{deg})\end{array}$ & \multicolumn{1}{c}{$N_{\text {vis }}$} \\
\hline $\mathrm{A}$ & 155 & 123 & 139 & 222750 \\
$\mathrm{~B}$ & 155 & 67 & 111 & 182250 \\
$\mathrm{C}$ & 155 & 4 & 79.5 & 141750 \\
$\mathrm{D}$ & 121 & 0 & 60.5 & 101250 \\
$\mathrm{E}$ & 59 & 0 & 29.5 & 60750 \\
$\mathrm{~F}$ & 3 & 0 & 1.5 & 20250 \\
\hline
\end{tabular}

Note. $-\Delta \alpha_{1}$ and $\Delta \alpha_{2}$ are the parallactic angle ranges at the central antenna (Pie Town) before and after source transit. The mean value is denoted as $\overline{\Delta \alpha}$. The number of visibilities in each simulated dataset is listed under column $N_{\text {vis }}$.

Table 2. Simulation source model component separations

\begin{tabular}{lrr}
\hline \hline & \multicolumn{1}{c}{$\Delta \alpha$} & \multicolumn{1}{c}{$\Delta \delta$} \\
Run code & $\begin{array}{c}\text { (mas) } \\
\text { (mas) }\end{array}$ \\
\hline $\mathrm{A}$ & -0.5 & +0.1 \\
\hline $\mathrm{X}$ & -0.25 & +0.05 \\
$\mathrm{Y}$ & -0.125 & +0.025 \\
$\mathrm{Z}$ & -0.0625 & +0.0125 \\
\hline
\end{tabular}

Table 3. Model-based bootstrap run parameters

\begin{tabular}{lcc}
\hline \hline Code & $\max \left(N_{p}\right)$ & $\Delta t_{b}(\mathrm{sec})$ \\
\hline M1 & 1 & 60 \\
M2 & 1 & 150 \\
M3 & 1 & 300 \\
M4 & 2 & 900 \\
\hline
\end{tabular}

Note. $-\max \left(N_{p}\right)$ is the maximum degree of the model polynomial used in each bootstrap model interval $\Delta t_{b}$. 
Table 4. Subsample bootstrap run parameters

\begin{tabular}{lc}
\hline \hline Code & $f_{s}$ \\
\hline S1 & 0.125 \\
S2 & 0.25 \\
S3 & 0.5 \\
S4 & 0.75 \\
\hline
\end{tabular}

Note. - $f_{s}$ is the fraction of data deleted in each subsample realization

Table 5. Bootstrap performance: variance scale factor $v_{f}$

\begin{tabular}{lllllllll}
\hline \hline Run code & M1 & M2 & M3 & M4 & S1 & S2 & S3 & S4 \\
\hline A & 1.00 & 1.00 & 0.99 & 1.02 & 0.16 & 0.33 & 0.77 & 1.93 \\
B & 0.95 & 0.99 & 1.01 & 1.03 & 0.19 & 0.34 & 0.78 & 1.94 \\
C & 0.97 & 1.02 & 1.00 & 1.03 & 0.20 & 0.38 & 0.91 & 2.05 \\
D & 0.98 & 0.98 & 0.98 & 0.99 & 0.19 & 0.43 & 0.91 & 1.90 \\
E & 0.96 & 0.98 & 0.95 & 1.01 & 0.28 & 0.52 & 1.16 & 2.37 \\
F & 0.91 & 0.94 & 1.03 & 0.98 & 0.29 & 0.48 & 1.02 & 1.70 \\
\hline X & 0.97 & 0.98 & 1.00 & 1.00 & 0.20 & 0.37 & 0.80 & 1.94 \\
Y & 0.96 & 1.00 & 1.01 & 1.02 & 0.19 & 0.37 & 0.79 & 1.92 \\
Z & 0.97 & 1.00 & 1.02 & 1.02 & 0.20 & 0.37 & 0.79 & 1.94 \\
\hline
\end{tabular}

Note. - The bootstrap performance measure $v_{f}$ is defined in the main text. 
Table 6. Bootstrap performance: variance mean-squared error $v_{M S E}$

\begin{tabular}{lccccccccc}
\hline \hline Run code & M1 & M2 & M3 & M4 & S1 & S2 & S3 & S4 & $\begin{array}{c}\min \left(v_{M S E}\right) \\
\times 10^{-15}\end{array}$ \\
\hline $\mathrm{A}$ & 1.42 & 1.05 & 1.00 & 1.07 & 3.18 & 2.53 & 1.73 & 1.52 & 3.40 \\
$\mathrm{~B}$ & 1.36 & 1.12 & 1.00 & 1.14 & 5.81 & 3.87 & 2.14 & 1.64 & 4.73 \\
$\mathrm{C}$ & 1.40 & 1.17 & 1.00 & 1.15 & 5.10 & 3.65 & 2.32 & 1.73 & 6.67 \\
$\mathrm{D}$ & 1.53 & 1.18 & 1.00 & 1.13 & 5.51 & 5.49 & 3.48 & 2.73 & 16.4 \\
$\mathrm{E}$ & 1.39 & 1.27 & 1.00 & 1.41 & 5.43 & 5.09 & 4.05 & 2.03 & 20.0 \\
$\mathrm{~F}$ & 2.30 & 1.47 & 1.00 & 2.28 & 14.8 & 12.3 & 10.7 & 7.94 & 985 \\
\hline $\mathrm{X}$ & 1.28 & 1.10 & 1.02 & 1.00 & 4.90 & 3.70 & 1.72 & 1.22 & 3.39 \\
$\mathrm{Y}$ & 1.45 & 1.35 & 1.17 & 1.00 & 5.40 & 4.49 & 2.16 & 1.51 & 2.91 \\
$\mathrm{Z}$ & 1.42 & 1.23 & 1.14 & 1.00 & 5.93 & 4.27 & 2.05 & 1.38 & 2.97 \\
\hline
\end{tabular}

Note. - The right-most column is the minimum $v_{M S E}$ measured across bootstrap type for each run code. The values for bootstrap codes $\{\mathrm{M} 1-\mathrm{M} 4, \mathrm{~S} 1-\mathrm{S} 4\}$ in each row are scaled by this minimum value, to allow the relative performance of the different bootstrap methods for a given run code to be more easily compared. 


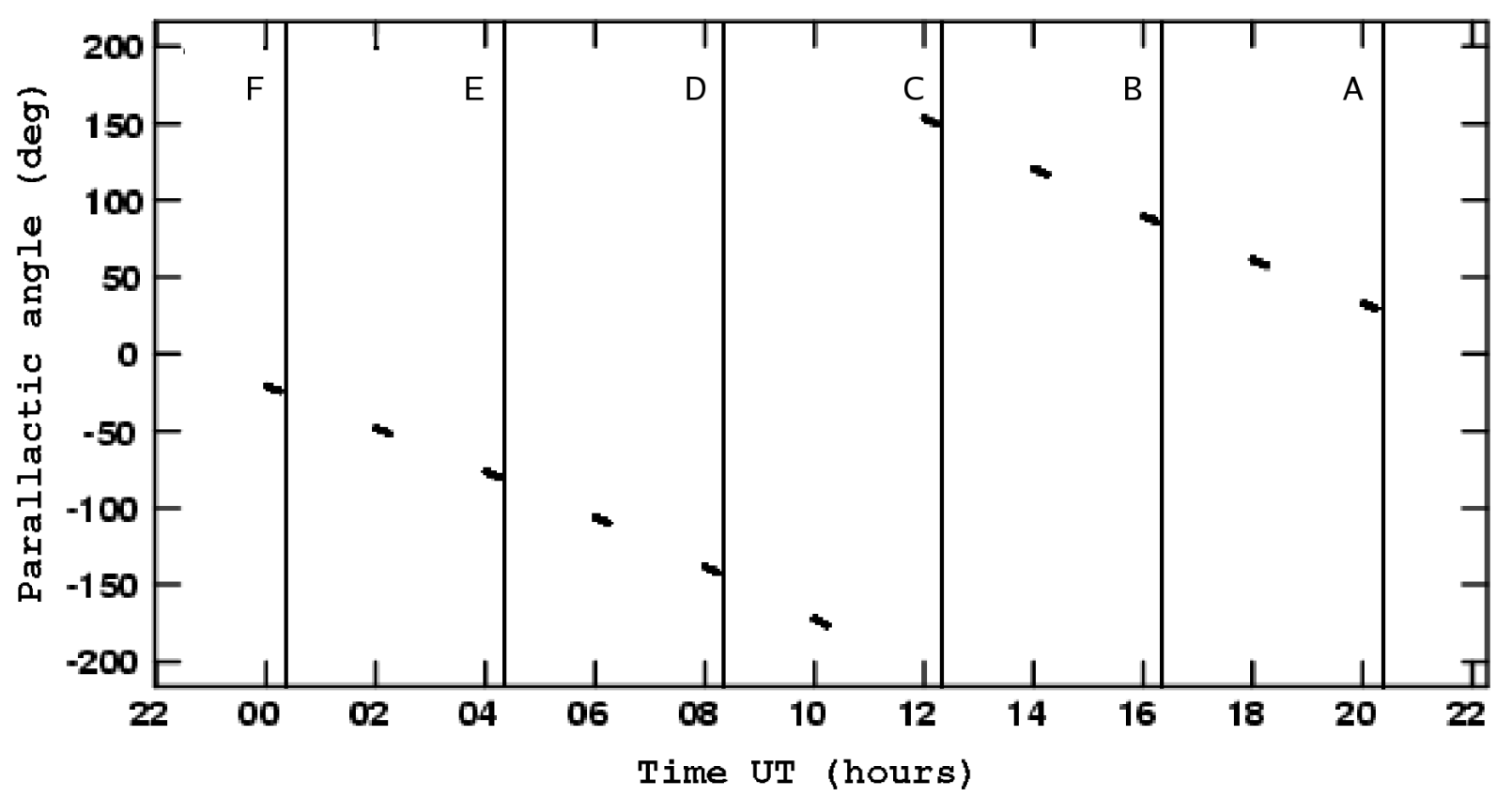

Fig. 1.- Parallactic angle variation at the central antenna (Pie Town) for the data used in the Monte Carlo simulations for run codes A-F, as enumerated in Table 1. Vertical lines denote the schedule truncation for each run code, as used to vary the parallactic angle range in the simulated data. 

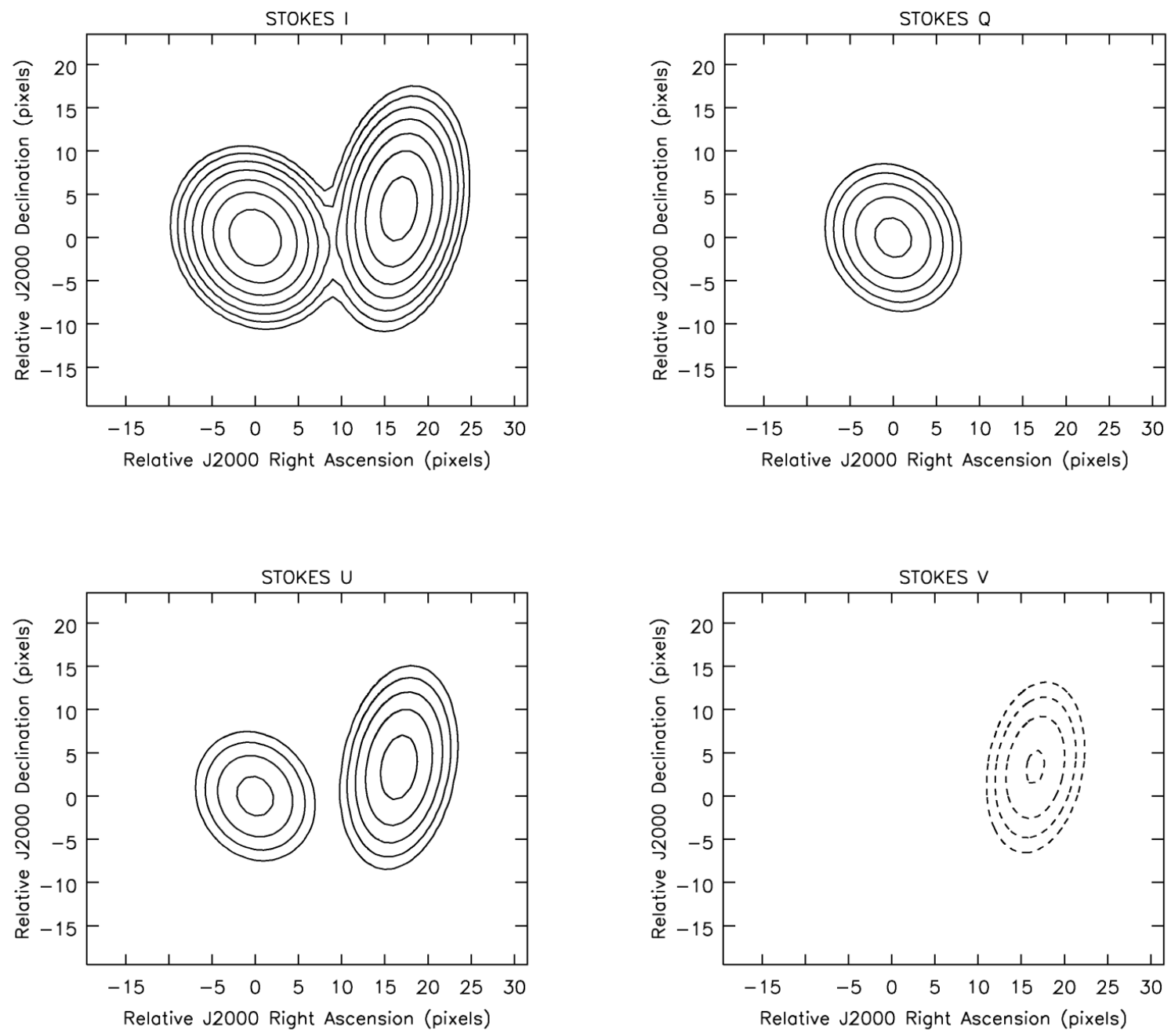

Fig. 2.- Simulation source model, convolved with a circular restoring beam of $156.007 \mu a s$, plotted in Stokes $\{I, Q, U, V\}$, using contour levels of $\{-64,-32,-16,-8,-4,-2,-1,1,2,4,8,16,32,64\} \times 4.434 \times 10^{-3}$ Jy per beam. The peak brightness in Stokes $\{I, Q, U, V\}$ is $4.434 \times 10^{-1}, 8.867 \times 10^{-2}, 9.591 \times 10^{-2}$, and $-3.836 \times 10^{-2}$ Jy per beam respectively. The source model consists of two polarized Gaussian components, denoted here by subscripts one and two, with parameters: $\left(\Delta \alpha_{1}=0, \Delta \delta_{1}=0\right), b_{m_{1} j_{1}}=0.2$ mas, $b_{\min _{1}}=0.15$ mas, P.A. ${ }_{1}=30 \mathrm{deg}, \mathrm{I}_{1}=1 \mathrm{Jy}, \mathrm{Q}_{1}=$ $0.2 \mathrm{Jy}, \mathrm{U}_{1}=0.1 \mathrm{Jy}, \mathrm{V}_{1}=0 \mathrm{Jy},\left(\Delta \alpha_{2}=-0.5, \Delta \delta_{2}=+0.1\right)$ mas, $\mathrm{b}_{\text {maj }_{2}}=0.3 \mathrm{mas}_{\mathrm{min} 2}=$ 0.1 mas, P.A.2 $=-10 \mathrm{deg}, \mathrm{I}_{2}=1 \mathrm{Jy}, \mathrm{Q}_{2}=0 \mathrm{Jy}, \mathrm{U}_{2}=0.25 \mathrm{Jy}, \mathrm{V}_{2}=-0.1 \mathrm{Jy}$. This Figure is reproduced from Figure 1 and Table 2 in Paper I. 


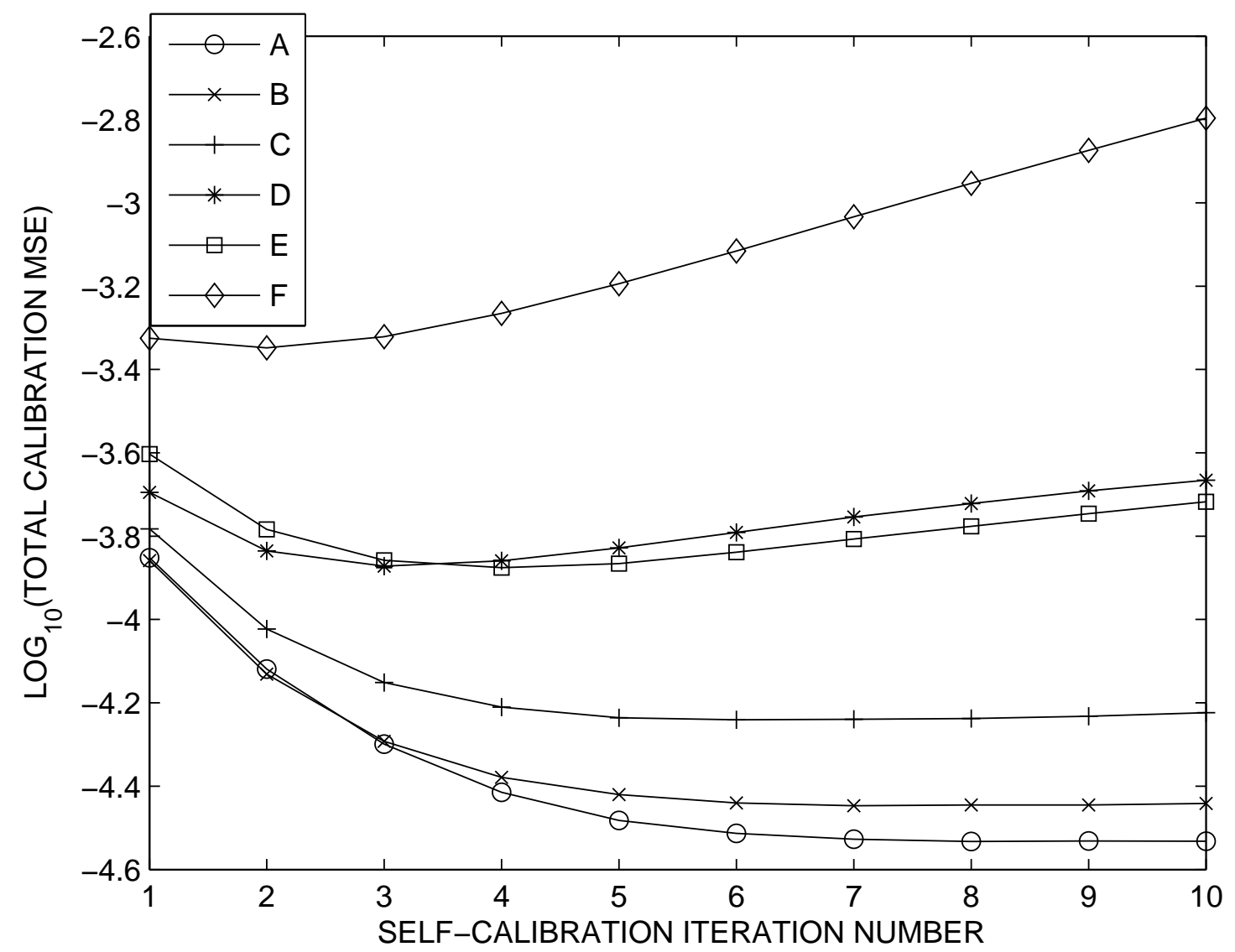

Fig. 3.- Total mean-squared error in the off-diagonal $D_{m}$ matrix elements, plotted for each run code $\{\mathrm{A}-\mathrm{F}\}$ against polarization self-calibration iteration number. As noted in Paper I, the total calibration MSE is computed here as: $\frac{1}{2 N} \sum_{p \in(R, L)} \sum_{m}^{N}\left(d_{m}^{p}-\breve{d}_{m}^{p}\right)\left(d_{m}^{p}-\breve{d}_{m}^{p}\right)^{*}$, where $d_{m}^{p}$ is the instrumental polarization determined by the solver, and $\breve{d}_{m}^{p}$ is the true value used to generate the simulated data. The decreasing parallactic angle ranges for run codes $\{\mathrm{A}-\mathrm{F}\}$ are listed in Table 1. 

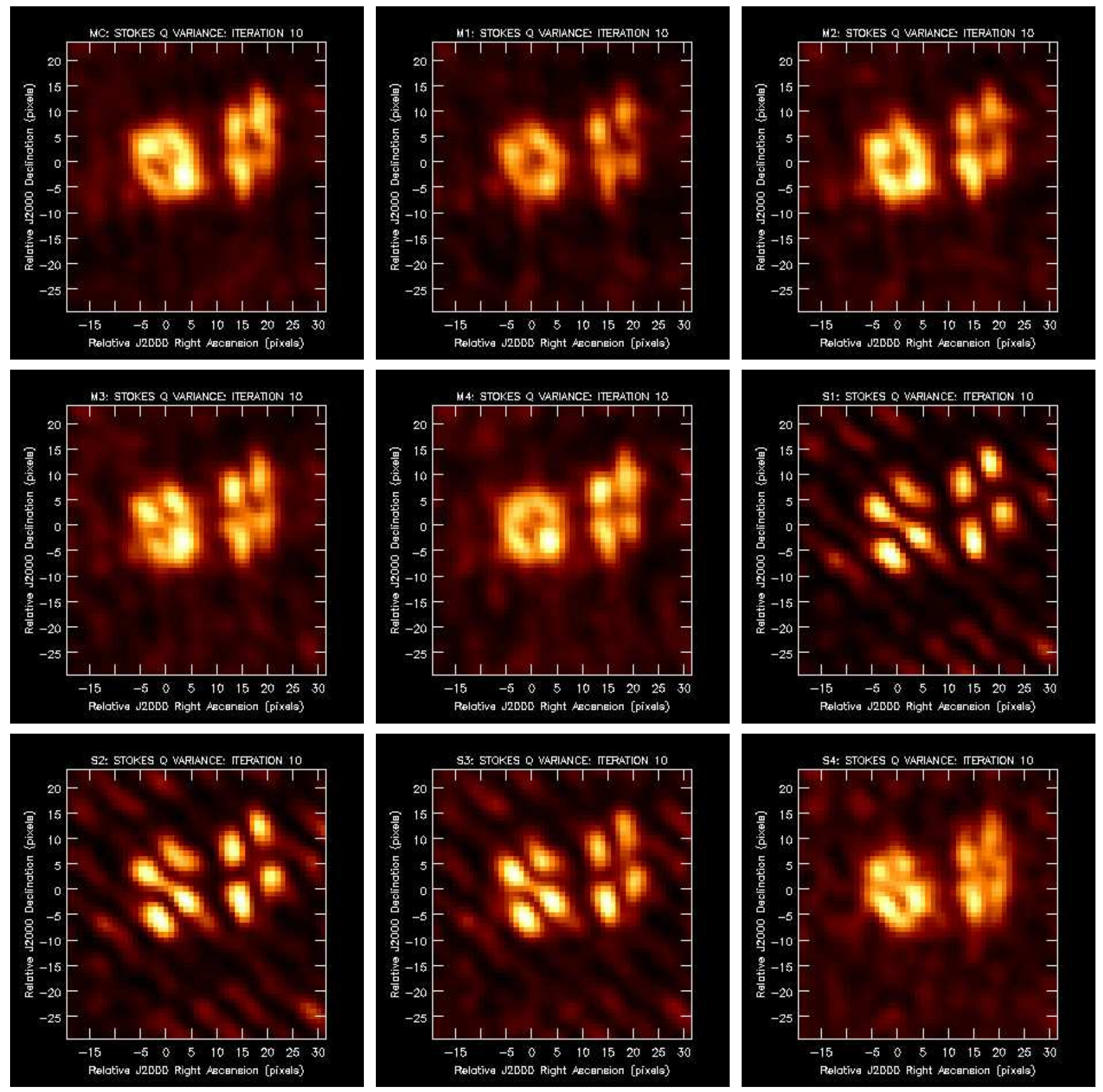

Fig. 4.- The imaging estimator Stokes $Q$ variance for the final iteration of polarization selfcalibration for run code B, as measured by direct Monte Carlo simulation (MC), model-based bootstrap resampling (M1-M4), and subsample resampling (S1-S4) methods(in sequential order from top left to bottom right). The parameters for these bootstrap resampling codes are listed in Table 3 and Table 4 respectively. The run code parameters are given in Table 1 and Table 2, The same default color mapping is used for each bootstrap image variance cube, where increasing color brightness denotes increasing image variance. This figure is included to allow morphological comparison of bootstrap variance images; a quantitative assessment of bootstrap performance is given in Table 6. See captions to Figures 8, 9, and 10 for quantitative variance ranges used in the associated contour plots. 

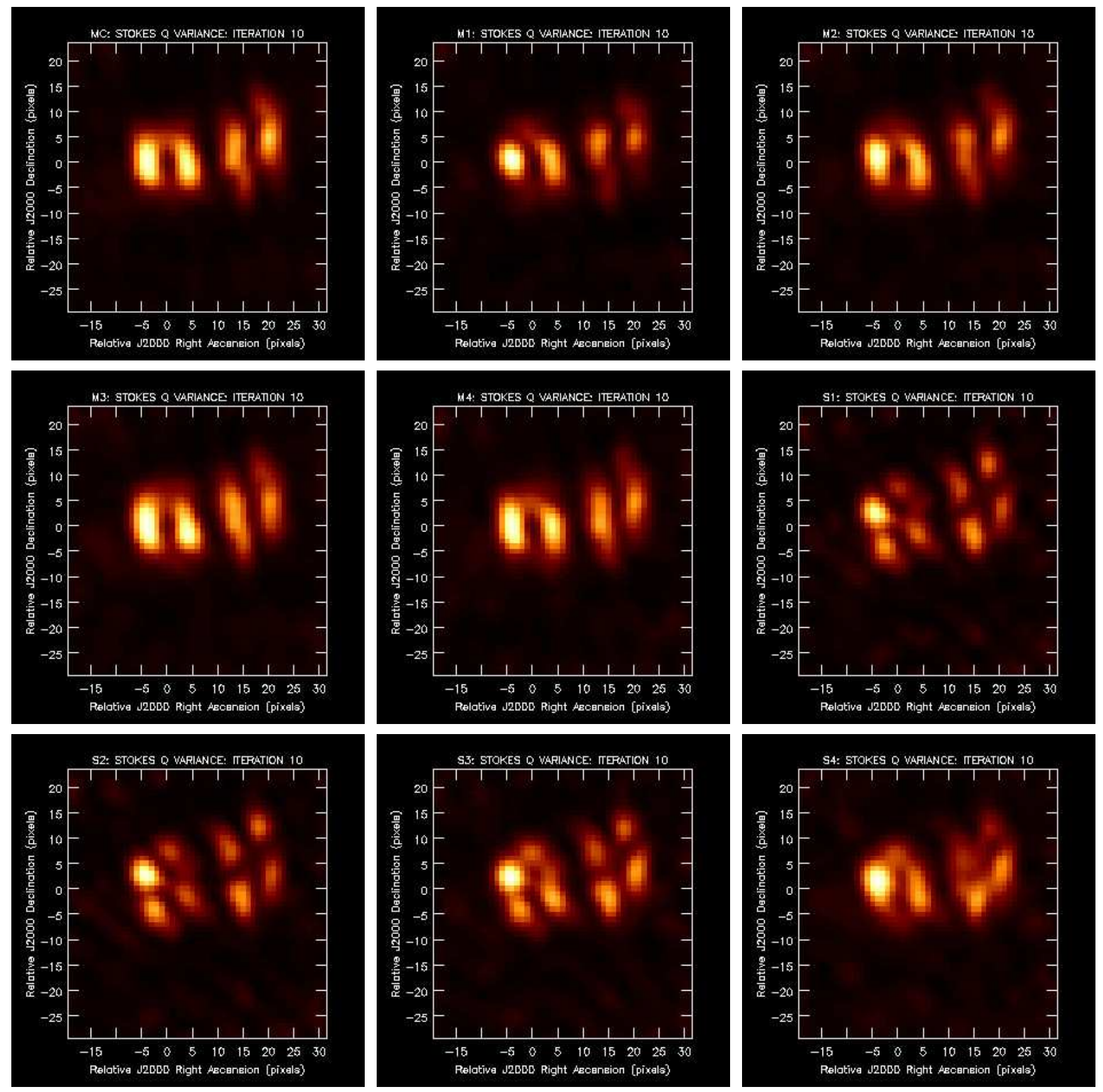

Fig. 5.- Same as Figure 4, but for run code D. 

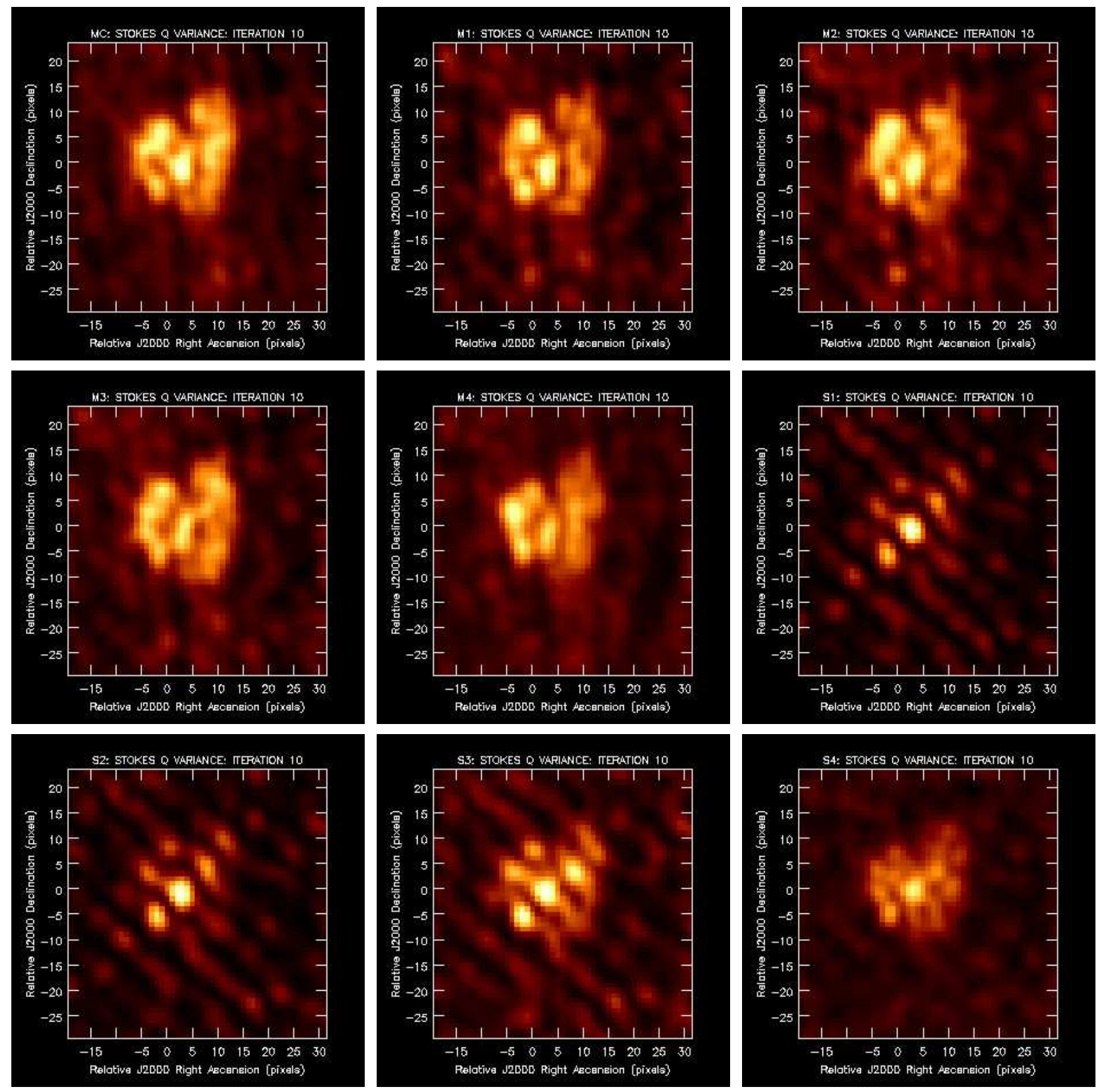

Fig. 6.- Same as Figure 5, but for run code X. 

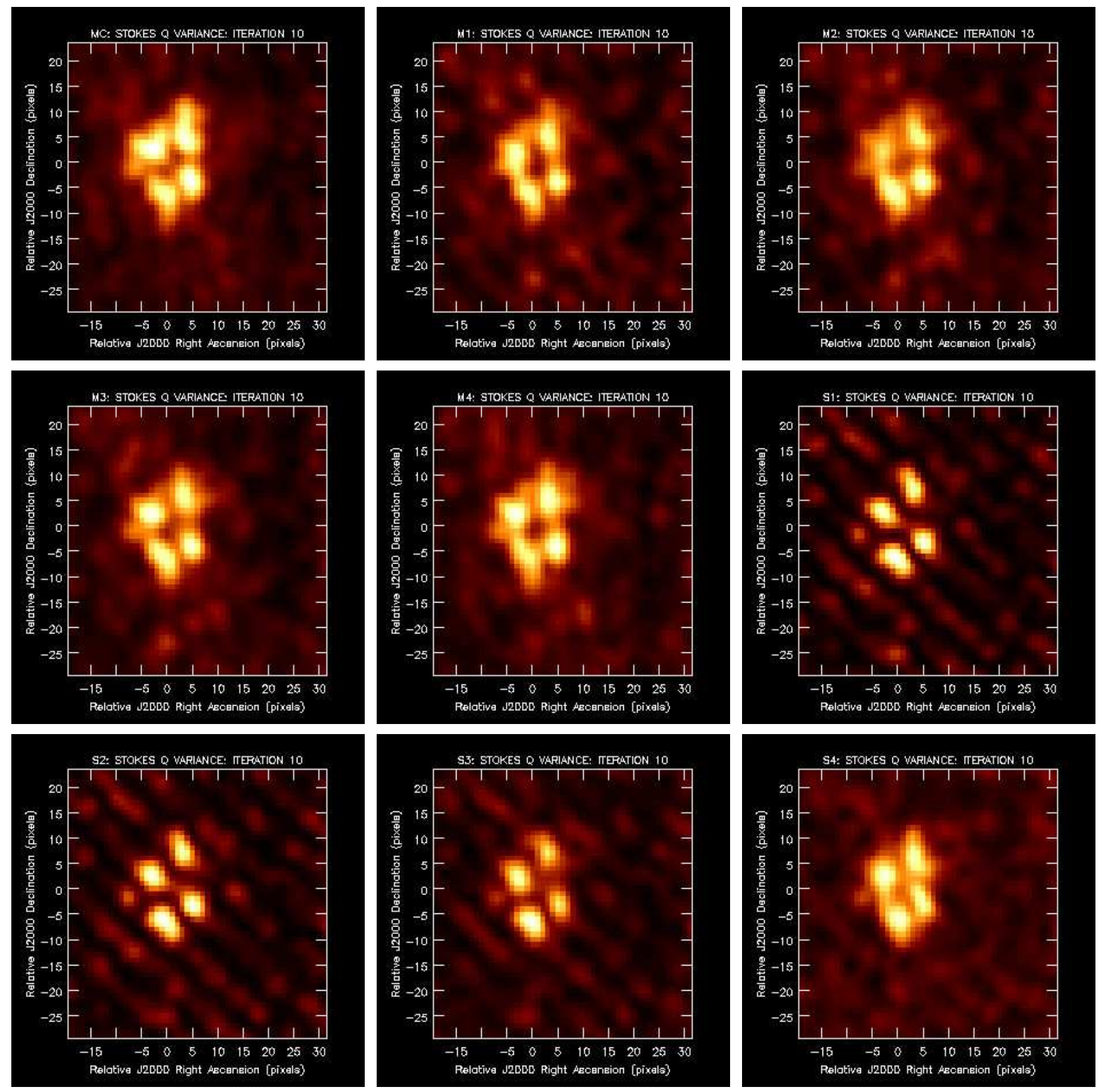

Fig. 7.- Same as Figure 6, but for run code Z. 

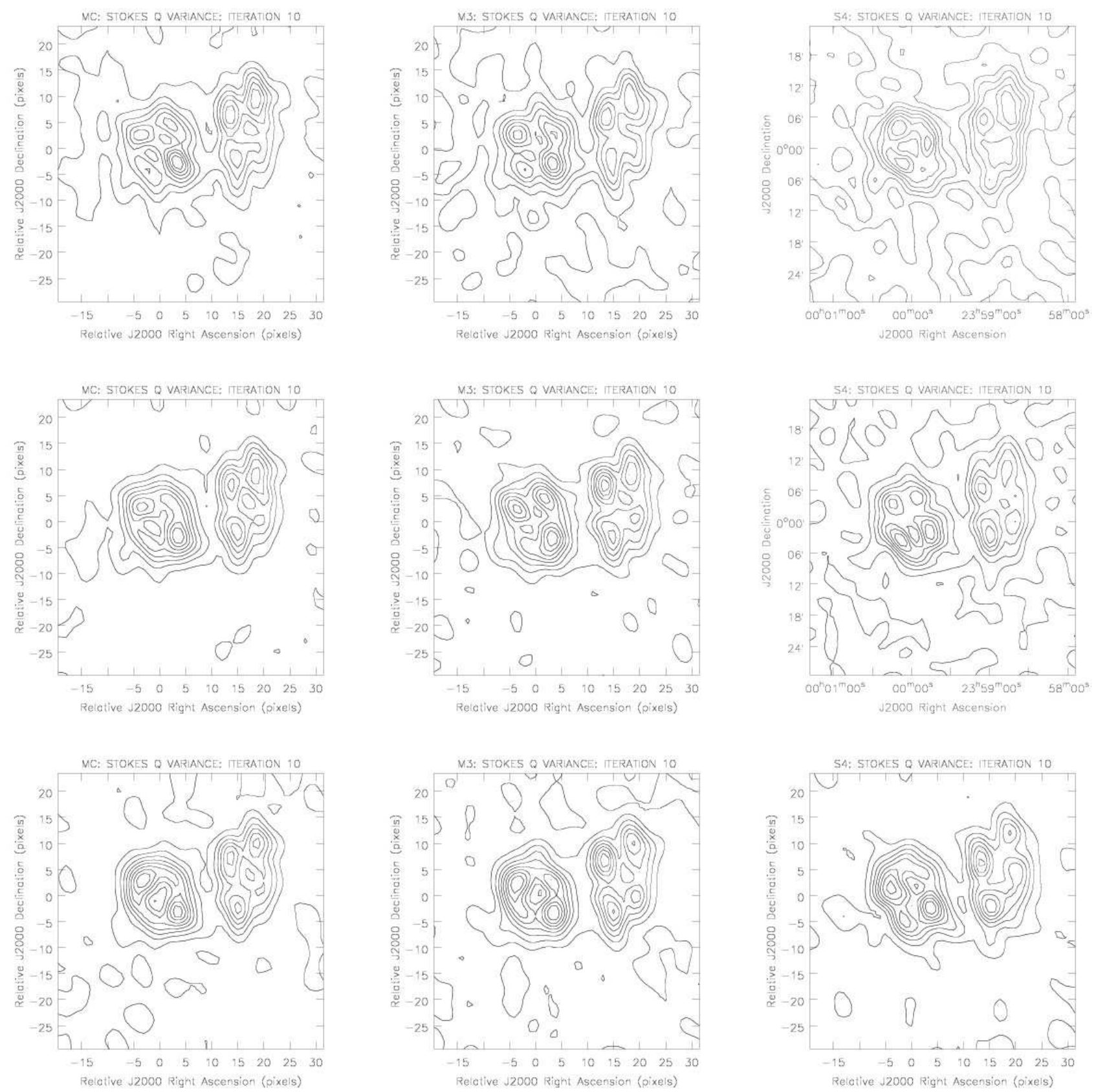

Fig. 8.- The imaging estimator Stokes $Q$ variance for the final polarization self-calibration iteration obtained by direct Monte Carlo simulation (MC; left), and for the optimum modelbased (M3; center column) and subsample (S4; right-most column) bootstrap methods identified in Table 6 for run codes A, B, and C (from top to bottom). The parameters for these bootstrap resampling codes are listed in Table 3 and Table 4 respectively. The run code parameters are given in Table 1 and Table 2. The contour levels are plotted at levels $\{0,0.1,0.2, \ldots, 1.0\}$ of the peak variance values: (A-MC: 1.883, A-M3: 1.783, A-S4: 1.924, B-MC: 2.534, B-M3: 2.429, B-S4: 3.089, C-MC: 3.697, C-M3: 3.450, C-S4: 5.580) $\times 10^{-6}$ $(\mathrm{Jy} / \text { beam })^{2}$. 

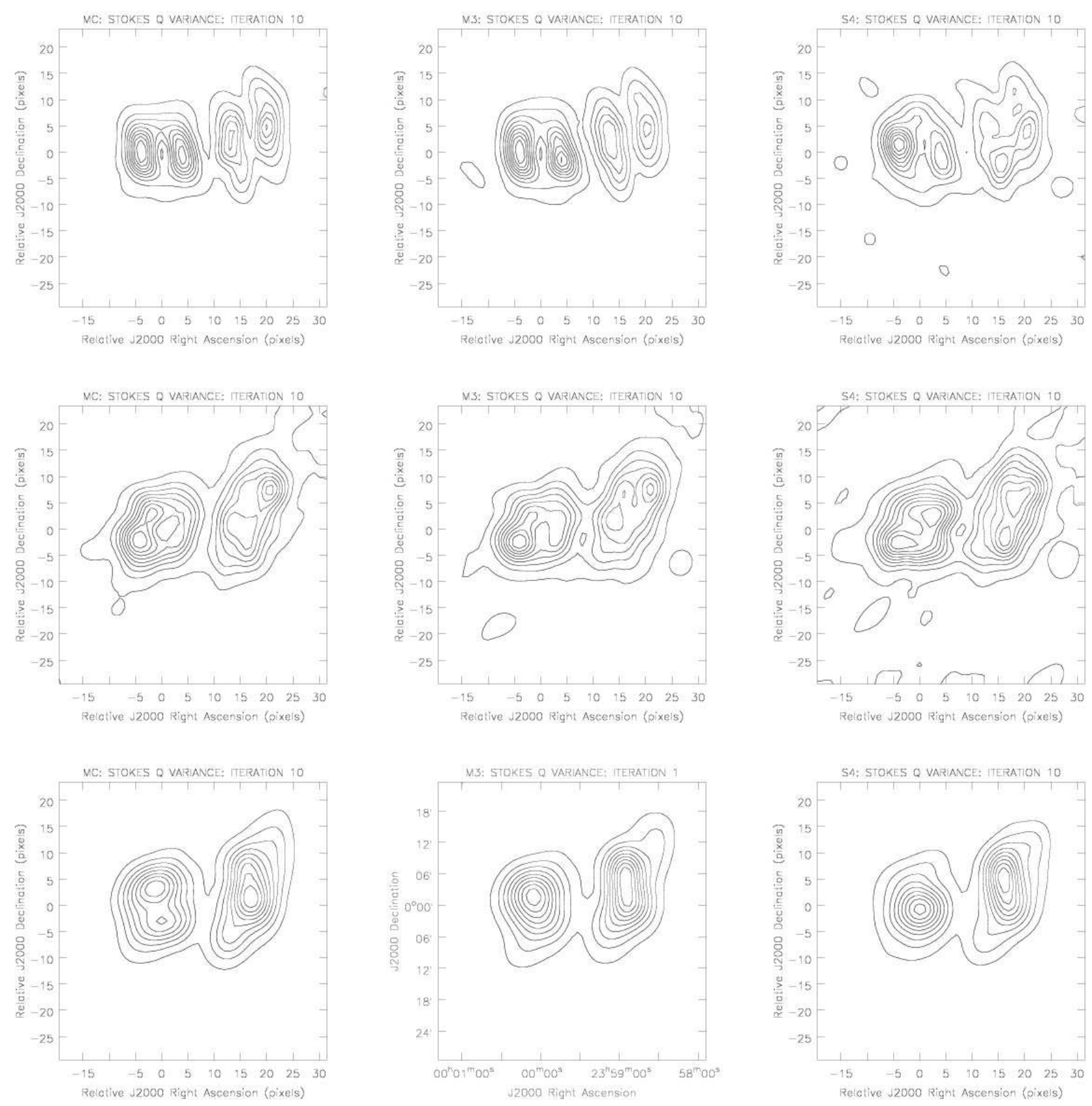

Fig. 9.- Same as Figure 8, but for run codes D, E, and F (from top to bottom), The contour levels are plotted at levels $\{0,0.1,0.2, \ldots, 1.0\}$ of the peak variance values: (D-MC: 12.74 , D-M3: 13.36, D-S4: 18.22, E-MC: 6.839, E-M3: 7.048, E-S4: 13.74, F-MC: 44.37, F-M3: 51.70, E-S4: 114.1$) \times 10^{-6}(\mathrm{Jy} / \text { beam })^{2}$. 

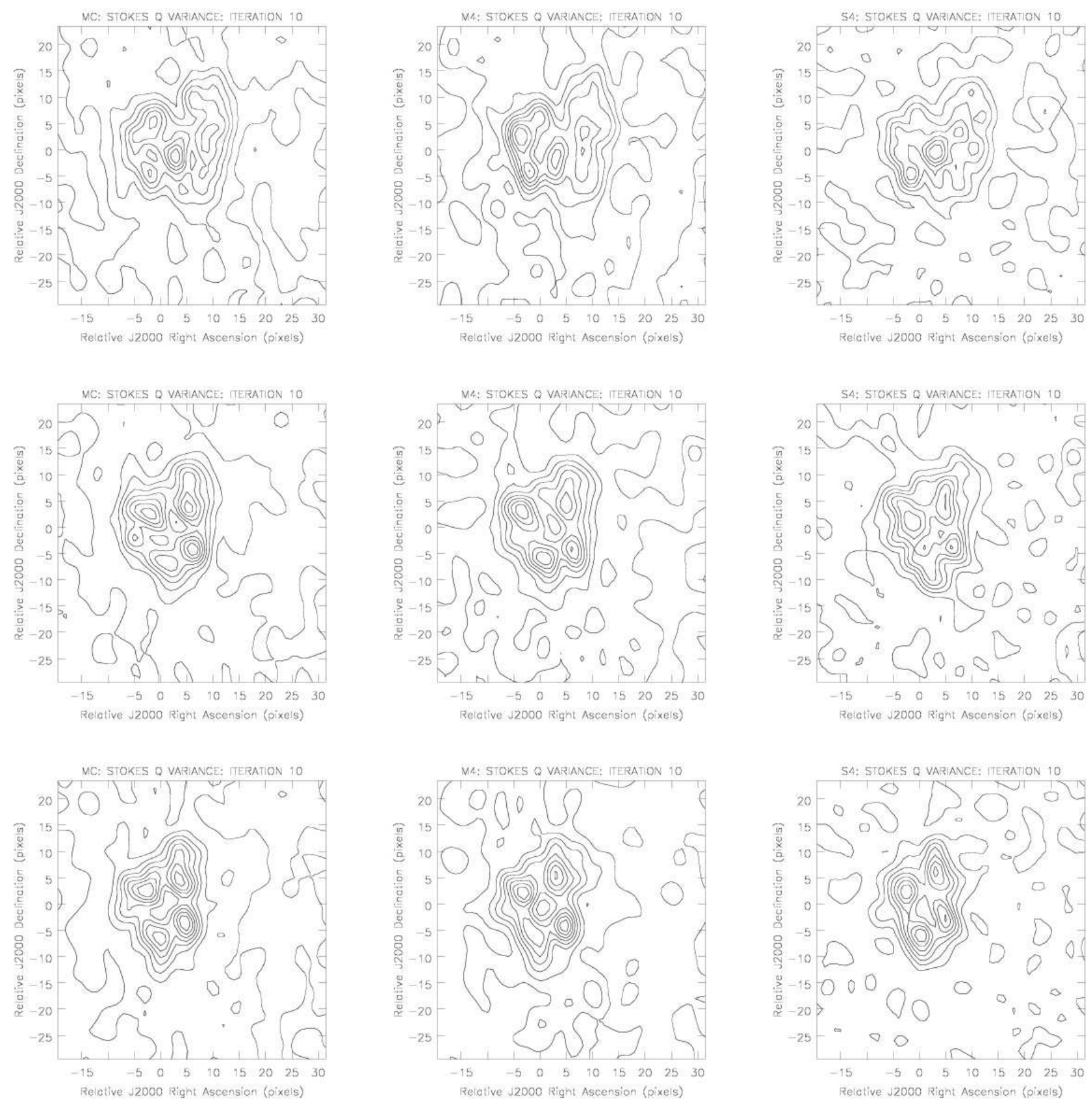

Fig. 10. - Same as Figure 8, but for run codes X, Y, and Z (from top to bottom), The contour levels are plotted at levels $\{0,0.1,0.2, \ldots, 1.0\}$ of the peak variance values: (X-MC: 1.451, X-M4: 1.372, X-S4: 2.415, Y-MC: 1.504, Y-M4: 1.438, Y-S4: 2.012, Z-MC: 1.507, Z-M4: $1.650, \mathrm{Z}-\mathrm{S} 4: 2.351) \times 10^{-6}(\mathrm{Jy} / \text { beam })^{2}$. 

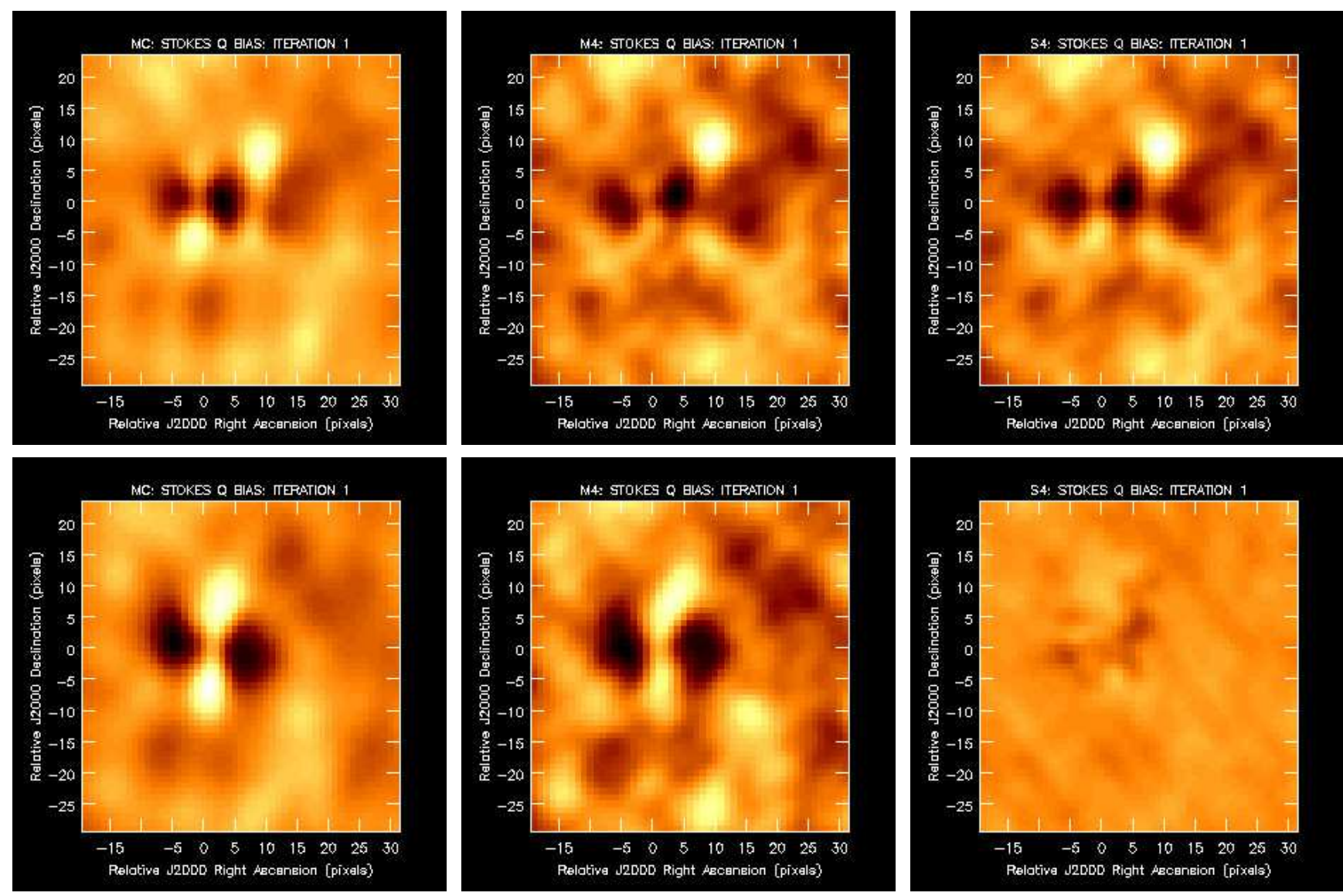

Fig. 11. - The imaging estimator Stokes $Q$ bias for the first iteration of polarization selfcalibration for run codes X (top) and Z (bottom), as measured by direct Monte Carlo simulation (MC; left), model-based bootstrap resampling (M4; center), and subsample bootstrap resampling ( $\mathrm{S} 4$; right) methods. The bootstrap codes are chosen for their optimality, as indicated in Table 6. The same default color mapping is used for each bootstrap image bias cube, where increasing color brightness denotes increasing image bias. 


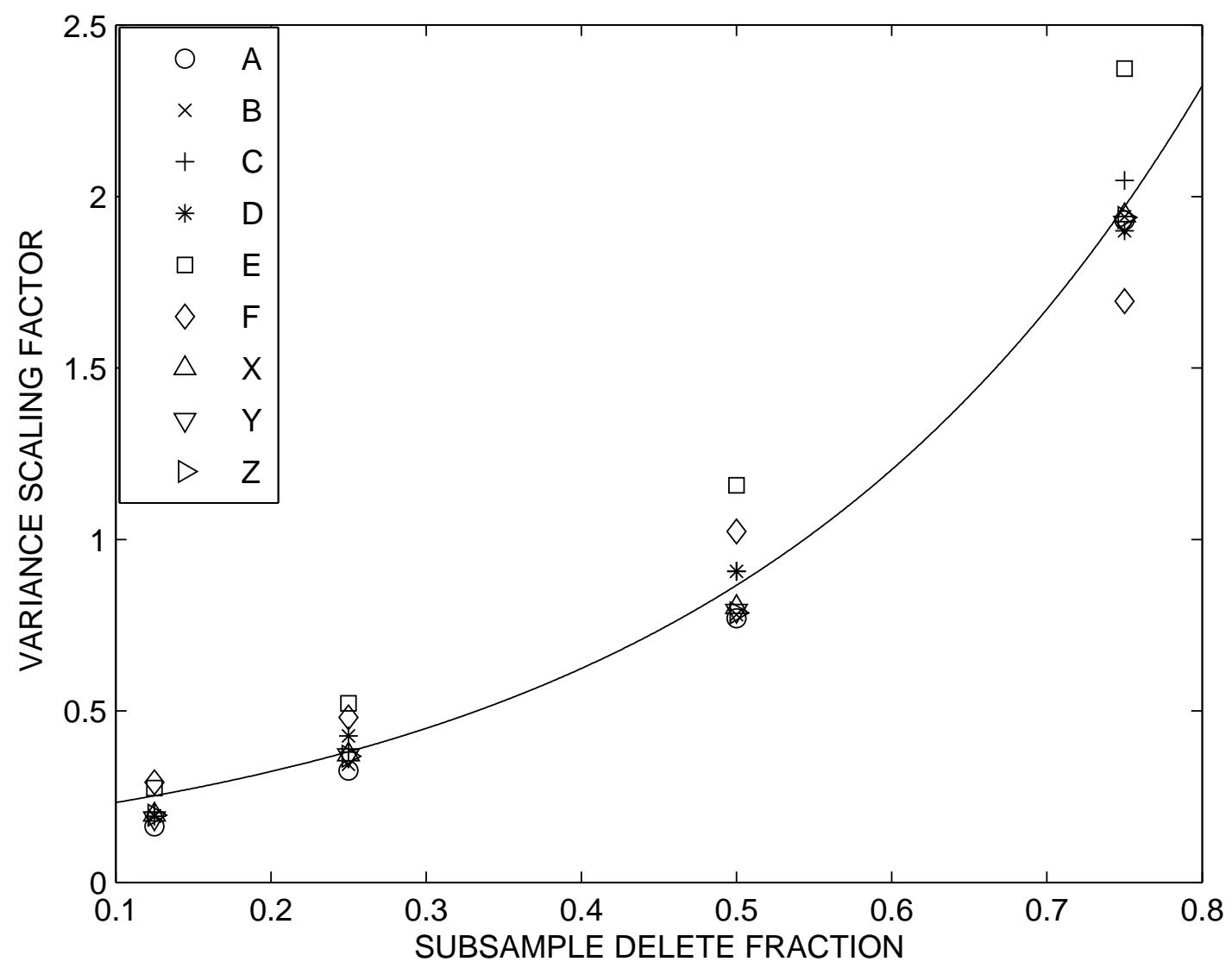

Fig. 12.- The variance scaling factor, $v_{f}$, plotted against the subsampling delete fraction, $f_{s}$, obtained from subsample bootstrap runs $\{\mathrm{S} 1-\mathrm{S} 4\}$ for run codes $\{\mathrm{A}-\mathrm{F}, \mathrm{X}-\mathrm{Z}\}$. The best joint exponential fit is plotted as a solid line, with parameters as described in the main text. 